\title{
What's the Chinese Food Like Here?
}

English version

\section{Shawn Wong}

\section{(2) OpenEdition Journals}

Electronic version

URL: http://journals.openedition.org/transtexts/167

DOI: 10.4000/transtexts. 167

ISSN: 2105-2549

Publisher

Gregory B. Lee

\section{Printed version}

Date of publication: 1 May 2006

Number of pages: $31-32$

ISSN: 1771-2084

\section{Electronic reference}

Shawn Wong, « What's the Chinese Food Like Here? », Transtext(e)s Transcultures 跨文本跨文化

[Online], 1 | 2006, Online since 13 September 2009, connection on 22 April 2019. URL : http:// journals.openedition.org/transtexts/167; DOI : 10.4000/transtexts. 167 


\section{What's the Chinese Food Like Here? by Shawn Wong}

I'm probably like most people—Ijudge a city by its Chinese food. Years ago, I added cappuchino to the list, but with the infestation of Starbucks everywhere on the planet, judging a town on whether or not they have both good Chinese food and good coffee proved irrelevant. The Chinese food standard still applies. It's an easy standard to apply because nearly every city in the world has a Chinese restaurant. Immigration theorists call it diaspora. I ain't no theorist. I just look for the Chinese restaurants. In the most remote towns, the Chinese restaurants have names of grandeur such as Jade Palace or Golden Riverside Inn (where there is no river) and these restaurants are usually decorated with lots of gold, red, and calligraphy proclaiming good luck and happiness while the tables are chipped laminate and vinyl covered chairs. If you can speak Chinese, you can usually talk to the owner of the restaurant into making you something real to eat instead of the usual items listed on the menu.

In Rome, the second most popular restaurants are Chinese restaurants. I'm afraid to eat Chinese food in Italy. I've peered into restaurants. I've seen the food sitting on the plates. I imagine there is good Chinese food here, but I also believe a lot of the food served in these place is some kind of elaborate act of revenge for Marco Polo stealing all that stuff from us. In Dingle, on the remote southwestern corner of Ireland, a little blond haired boy stares at me in the grocery store. I know why. I say, "Hello." Now he's wide-eyed. His mother replies for the suddenly mute boy. I'm still holding the experience of driving down to Dingle over Connor Pass in a fog so dense, I had to crane my head out the window of the car so that I could drive parallel to the stones lining the road on my right. What does the boy see? He sees an Asian person where there are none. I hear water. Am I driving toward a river ? Is the road washed out? I let the car coast slowly toward the sound until I know I'm right at the edge of the water. There's still a road, but there's a waterfall cascading from a rock above the road and it empties out onto the middle of the road. As soon as I pass underneath it- the water washing over the car-the fog breaks and I stop the car. The fog bank is really a cloud and when I get out of the car, the bottom of the cloud is just a few feet above my head. The Irish poet, Nuala Ni Dhomhnaill warned this would happen. Rather than give me directions to Dingle, she listed a series of images-the narrow stone-lined road, the waterfall, the expansive view of the valley below on the descent from Connor Pass, her grandmother's house on the coast of Dingle by the ocean. Later, she sends me a picture of herself as a young woman holding her baby daughter. Perhaps it's the same way I communicate-through images and the past. The mother of the little boy pulls him away to divert his gaze. She whispers, "Sorry," to me. I should tell the boy that I'm Chinese so that he can complete the story that's unfolding in his head. In another part of the world, a woman who owns a laundry in Bellagio, Italy doesn't 
ask me for my name when I leave my clothes to be cleaned. When I pick up the clothes, she's pinned the word, "cinese," inside the waistband of one of my pants. The next time, she doesn't bother to label my clothes. I'm the only Asian person, let alone the only Chinese person, living in the town.

In Germany, you can tell where a person is from by looking at the license plate of his or her car- “M" for Munich, "B" for Berlin, "Tü" for Tübingen. When I lived in Tübingen for several months, several people stopped me and pointed at my red license plates and asked where I was from and many, many people stared at the plates first, then up at me. I could see they were puzzled. An Asian person driving a car with unidentifiable license plates. The car and the license were from Sweden and I was not. In Tübingen I never saw a house for sale and one woman who was from a small town near Tübingen said that in her entire life she could not remember anyone moving away from her hometown.

In Rome, late one Sunday night, I get into a taxi and I ask the driver, in Italian, to take me to the nearest open pharmacy. After driving for a minute or two, he says me in an language that I know isn't Italian. I'm not sure I heard him correctly, so I answer, "Che ?" He asks me again and this time there's no mistaking that he asked me if I speak Japanese in Japanese. I tell him, in Italian, that I'm not Japanese and that I'm American. He can't believe I'm not one of those ubiquitous Japanese tourists. I resign and tell him I'm cinese from America. "Ah, OK, Rambo," he replies. I think l'd rather be Japanese than named after a Sylvester Stallone character. A few minutes later, he asks me, "Nee how ma ?" in Chinese. I tell him I speak better Italian and German than Chinese. We speak German for the rest of the trip, but he still calls me Rambo.

In 2001, I was invited to a conference at Hong Kong University to be an example of the diaspora. The university invited writers of Chinese ancestry from all over the world who wrote poetry or fiction in English. There were probably two dozen of us gathered in Hong Kong to discuss the diasporic issues that surround us and our work. One afternoon between serious sessions I was drinking a beer with novelist Timothy Mo from the UK and Hsu-Ming Teo, a young novelist from New Zealand. This was the moment the conference organizers were hoping would happen. If a literary scholar were seated with us, I'm sure his or her theory quill would have been quivering with anticipation above the ink well. Neither Timothy nor I had read Hsu-Ming Teo's novel, Love and Vertigo, so we asked the question two well-published and seasoned writers would ask at a conference, "What's your book about ?" Hsu-Ming sighed, shrugged, and replied, "Family and identity." We laughed and ordered more beer. End of literary conversation. The family and identity book is a rite of passage for all of us and it didn't matter where the borders were set or where we went to school or what language we used. I took a sip of beer and asked Timothy, who was born in Hong Kong, "So, you know a good Chinese restaurant here ?" 


\section{La cuisine chinoise est-elle bonne dans le coin?}

Comme la plupart des gens, je juge une ville à la qualité de sa cuisine chinoise. Jadis, j'avais ajouté le cappuccino à la liste, mais vue l'épidémie de Starbucks partout sur la planète, il est devenu impossible de juger une ville à la qualité de sa cuisine chinoise et de son café. Pour la cuisine chinoise, ça marche toujours, et c'est un critère facile à utiliser puisque pratiquement toutes les villes du monde ont un restaurant chinois. Les théoriciens de l'immigration appelle cela la diaspora. Moi, je ne suis pas théoricien. Je ne fais que rechercher les restaurants chinois. Dans les coins les plus reculés, ils ont des noms grandioses comme le Palais de Jade ou l'Auberge de la Rive Dorée (sans rivière) et sont souvent ornés d'un foisonnement d'or, de rouge et de calligraphie proclamant bonheur et heureuse fortune, alors que leurs tables sont en contreplaqué écaillé et leurs chaises en vinyle. Si vous parlez chinois, vous pouvez toujours convaincre le propriétaire de vous cuisiner quelque chose de vraiment comestible au lieu des plats habituels figurant au menu.

A Rome, les restaurants chinois tiennent la deuxième place au palmarès de la popularité. J'ai peur de manger chinois en Italie. J'ai jeté un coup d'œil dans les restaurants. J'ai vu la nourriture dans les assiettes. Je pense qu'il est sans doute possible de trouver de la bonne cuisine chinoise ici, mais je suis aussi persuadé que la nourriture que l'on sert dans ces endroits est en quelque sorte un acte de vengeance contre Marco Polo qui nous a tout piqué.

Dans l'épicerie, à Dingle, au fin fond du Sud-ouest de l'Irlande, un petit garçon blond me fixe du regard. Je sais pourquoi. Je lui dis : «Hello». Ses yeux s'écarquillent de plus belle. Sa mère répond pour l'enfant frappé soudain de mutisme. Je suis toujours plein du souvenir de mon arrivée à Dingle par le col de Connor dans un brouillard si épais, que j'avais dû passer la tête par la fenêtre pour arriver à longer les pierres qui bordaient la route sur ma droite. Que voit le petit garçon? I| voit un Asiatique dans un endroit où il n'y en a pas. J'entends un bruit d'eau. Suis-je près d'une rivière ? La route a-t-elle été emportée ? Je laisse la voiture glisser doucement vers le bruit, mais soudain une chute d'eau déferlant de rochers surplombant la route se déverse en plein milieu. Une fois la chute franchie - l'eau s'abattant sur le capot de la voiture - le brouillard s'éclaircit et je m'arrête. La nappe de brouillard est en réalité un nuage et quand je sors de la voiture, il se trouve juste au-dessus de ma tête. La poétesse irlandaise Nuala Ni Dhomhnaill avait évoqué ce phénomène. Plutôt que d'indiquer la route menant à Dingle, elle avait dépeint une série d'images - la route étroite bordée de pierres, la chute d'eau, l'imposante vue de la vallée en descendant du col de Connor, la maison de sa grand-mère sur la côte de Dingle au bord de l'océan. Plus tard, elle m'envoya une photo d'elle, jeune, tenant dans ses bras sa fille bébé. C'est peut-être ainsi que je communique 
aussi - par les images et le passé.

La mère du petit garçon l'entraine pour l'arrêter de bader. Elle me murmure : «Désolée ». Je devrais dire au petit garçon que je suis chinois pour qu'il puisse terminer l'histoire qu'il est en train de se raconter. Ailleurs dans le monde, une femme qui tient une blanchisserie à Bellagio, en Italie ne me demande pas mon nom quand je lui laisse mes vêtements à nettoyer. Quand elle me les rend, je remarque, épinglé à l'intérieur de la ceinture d'un de mes pantalons, le mot «cinese ». La fois suivante, elle ne marque même plus mes vêtements. Je suis le seul Asiatique, le seul Chinois de la ville.

En Allemagne, vous pouvez savoir d'où vient quelqu'un en regardant la plaque minéralogique de sa voiture - « $M$ » pour Münich, «B » pour Berlin, «Tü » pour Tübingen. Pendant mon séjour de quelques mois à Tübingen, plusieurs personnes m'ont arrêté dans la rue et m'ont demandé d'où je venais en indiquant mes plaques d'immatriculation rouges, et nombreux étaient ceux qui me regardaient puis regardaient les plaques. Je voyais bien qu'ils étaient perplexes. Un Asiatique conduisant une voiture aux plaques minéralogiques inconnues. La voiture et les plaques venaient de Suède mais pas moi. A Tübingen, je n'ai jamais vu de maison à vendre et une femme qui venait d'une petite ville des environs m'a dit que de toute sa vie personne n'avait déménagé de sa ville natale.

A Rome, un dimanche soir, tard, je prends un taxi et je demande au chauffeur, en italien, de m'amener à la pharmacie la plus proche. Après quelques instants, il s'adresse à moi dans une langue que je sais n'être pas de l'italien. Comme je ne suis pas sûr d'avoir bien entendu, je lui demande «Che ? ». II pose de nouveau sa question et cette fois-ci, j'en suis sûr, il me demande en japonais si je parle japonais. Je lui répond en italien que je ne suis pas japonais mais américain. II n'arrive pas à croire que je ne suis pas un de ces touristes japonais omniprésents. De guerre lasse, je lui dis que je suis un cinese d'Amérique. «Ah, je vois, Rambo » répond-il. Je crois que j'aime mieux être pris pour un Japonais que pour un personnage incarné par Sylvester Stallone. Quelques instants plus tard, il me demande « Ni hao ma ? " en chinois. Je lui dis que je parle mieux italien et allemand que chinois. Nous parlons allemand le reste de la course, mais il continue à m'appeler Rambo.

En 2001, j'étais invité en tant qu'exemple de la diaspora à une conférence à l'Université de Hong Kong. L'université avait invité des écrivains du monde entier dont les ancêtres étaient chinois et qui écrivaient de la poésie et des romans en anglais. Nous devions être deux douzaines à discuter des préoccupations diasporiques nous concernant ainsi que notre travail. Un après-midi, entre deux sessions, je buvais une bière avec Timothy Mo du RoyaumeUni et Hsu-Ming Teo, un jeune romancier de Nouvelle-Zélande. C'était l'instant tant attendu des organisateurs de la conférence. Si un chercheur théoricien de la littérature avait été des nôtres à ce moment-là, je suis certain que sa plume aurait frémi d'impatience dans l'encrier. Ni Timothy ni moi n'avions lu Love and Vertigo, le roman de Hsu-Ming Teo, et nous lui avons posé la question habituelle de deux écrivains chevronnés lors d'une conférence : «De quoi parle ton roman? » Hsu-Ming a soupiré puis a répondu, dans un haussement d'épaules : "De famille et d'identité ». Nous avons ri et commandé une autre bière. Fin de conversation littéraire. Le livre sur la famille et l'identité est un rite de passage pour nous tous, peu importe où il se déroule, où nous sommes allés à l'école ni quelle langue nous utilisons. J'ai bu une gorgée de bière et j'ai demandé à Timothy, qui est né à Hong Kong : «Alors, tu connais un bon restaurant chinois ici ?» 


\section{此地中 \\ 国食物如何?}

可能像大部分人一样, 我评判一个城市的标准是该城市的中国食物。几年 前, 我曾经把卡布奇诺咖啡(Cappuchino)也算作评判标准之一。但是随着星巴克 (Starbucks) 在全球各地开枝散叶, 把美味的中国食物和香醇的咖啡拉到一块, 作为评判一个城市的标准显得有点不伦不类。但是中国食物这条标准依然奏 效。这条标准其实应用起来相当便利, 因为世界上几乎每一个城市都有中餐 馆。移民理论家们称之为 “离散”。但是我不是什么理论家, 我只是寻找中餐 馆而已。在世界最边远的城镇里, 中餐馆的名字大多气派非凡, 像什么玉殿、 黄金河岸旅馆（其实旁边根本没有河）之类。这类餐馆墙上一般挂了许多金黄 色和红色的书法大字作为装饰, 象征幸福好运等等。但是餐馆的桌椅一般是层 压板、乙烯塑料制成。如果你能讲中文, 你大可以说服餐馆老板给你做顿真正 的饭, 而不是给你端来菜牌上列的东西。

中餐馆在罗马是第二受欢迎的。我很怕在意大利吃中国食物。我曾经窥见过 餐馆里的情形, 食物堆在盘子里的样子我还记得。我相信意大利有好的中餐 馆, 但是我也相信, 这里中餐馆的食物, 是对罗马人的一种报复, 报复当年马 可・波罗偷取了我们许多东西。

丁格尔（Dingle）位于爱尔兰西南部一个边远角落。在那儿的杂货店里, 有 个金发小男孩死盯着我看。我知道他好奇些什么。我跟他打声招呼: “你 好! ”他吃了一惊, 瞪大眼睛, 说不出话来。他妈妈只好代他回应。我还记得 从康诺尔山口 (Connor Pass) 开车去丁格尔的情形。那天正好大雾, 我不得不 从车里探着脑袋看路, 以免车子撞上马路右边的石头。那小男孩看见什么了? 他看见了他们那地方唯一的一个亚洲人。我听见水流声了。我是不是朝一条河 开去? 马路是不是被水冲走了? 我任由车子向水声传来的方向滑去, 直到我觉 得车子已经到了水边为止。前面还有路, 但是马路上方一块岩石上, 一道瀑布 倾泄而下, 水全涌到了马路中间来。我开车从瀑布下经过, 水流冲刷着车子。 车子过了瀑布后浓雾已散, 我把车子停了下来。雾气尽头飘着一朵云层, 我 从车里出来, 云层底部离我的头顶只有几英尺而已经。爱尔兰诗人多姆奈尔 （Nuala Ni Dhomhnaill）早跟我说过会看见这种景象。我要去丁格尔, 她不是给 我指路, 而是给我开了张单子, 上面列了一系列的景象：两边排满石头的窄 路、瀑布，从康诺尔山口的坡上下来可以看见的广阔的峡谷，还有她祖母在丁 格尔海岸边的一座小房子。后来她给我寄了张照片, 照片上的她很年青, 抱着 自己的女儿。我想这或许也是我交流的方式：通过意象和过去。

那小男孩的妈妈推了他一把, 他的视线移开了。她对我轻轻地说了句: “对不 起”。我应该告诉那小男孩我是中国人, 这样才能让他给脑海里展开的故事找 个好结局。在意大利的碧堤半岛, 我去洗衣店洗衣服的时候, 女店主没有问我 的名字。我去取回衣服的时候, 她在我的一条裤子的腰带上别了张条, 上面写 着“中国人”(Cinese)。后来我再去, 她都没有在我的衣服上别条子了。因为我 不仅是那小镇上唯一的中国人, 而且还是那儿唯一的亚洲人。

在德国，车主的籍贯是通过车牌字母表现的。“M”代表慕尼黑（Munich），“B”代 
表柏林（Berlin），“Tü”代表蒂宾根（Tübingen）。我在蒂宾根住过几个月。 那段时间里, 有好多人曾经拦过我的车。他们指着我的车牌问我从哪里来。还 有好多人先是看看车牌, 然后又抬起眼睛看看我。我明白他们的疑惑, 一个亚 洲人驾着一辆来历不明的车。那辆车和车牌来自于瑞典, 但我不是。在蒂宾根 从来没看见过有房屋出售。旁边小镇的一个女人告诉我, 她这辈子, 没见过谁 离开过那个小镇。

一个周日晚上, 我在罗马上了一部出租车。我用意大利语告诉司机去最近的一 家药店。开车一两分钟后, 司机用一种绝对不是意大利语的语言跟我讲话。我 不知道自己有没有听错, 只好说: “什么?”他又问了我一遍, 这次我终于听 清楚了。他用日语问我是不是日本人。我用意大利语告诉他说, 我不是日本 人, 我是美国人。他根本不相信我, 因为这地方到处都是日本游客。最后我终 于死心了, 我告诉他, 我是一个从美国来的中国人(cinese)。他说: “噢, 明白 了, 是兰博 (Rambo) ”。我想我宁愿当日本人, 也不愿意被人用史泰龙 (Sylvester Stallone) 电影里面的一个人物的名字来称呼。几分钟以后，他又用 中文问我: “你好吗?”我告诉他我的意大利语和德语比中文要好。最后我们 一直就用德语交谈, 但是他一直都叫我兰博。

2001年，我以离散作家的身份，受邀到香港大学参加一个会议。受邀参加会议 的人是世界各地用英语写作而且拥有中国血统的作家。大约有二十多名与会者 聚集在香港，讨论我们和我们工作周围的离散问题。有一天的会议休息时间， 我和英国的小说家茅（Timothy Mo），还有新西兰的年轻小说家图（Hsu-Ming Te0）一起喝啤酒。这一刻正是会议组织者最希望看见的。如果一位文学学者 跟我们坐在一起的话, 我想他的灌满了理论的羽毛笔管会因为激动而在墨水瓶 上发抖。我和茅（Timothy Mo）都没有读过图（Hsu-Ming Teo）的小说《爱情和军 眩》，于是我们问了一个畅销作家在会议上可能会提的问题： “你的书是关于 什么的?”图（Hsu-Ming Teo）叹了口气，䇤年肩说：“家庭和身份”。我们大 笑, 然后点了更多的啤酒。这就结束了我们的文学对话。那本关于家庭和身份 的书, 对于我们来说是一种谈话开始的仪式。故事发生的国界, 我们在哪儿上 学或者我们使用什么语言都不重要。我嗓口啤酒, 问出生在香港的茅 (Timothy Mo）：“你知道这里哪家中餐馆比较好吗? 